\title{
Assessment of the relationship between the morphology of the cervical spine and Class II population by CBCT
}

\author{
Ana Natália de Oliveira (IC), Gina Delia Roque-Torres (PG), Priscila Peyneau (PG), Solange Maria de \\ Almeida Bóscolo (PQ), Frab Norberto Bóscolo (PQ), Glaucia Maria Bovi Ambrosano (PQ).
}

\begin{abstract}
Associations were found between fusions of the cervical column and mandibular retrognathia, large cranial base angle, and large horizontal overjet. The objective of this study was to evaluate the relationship between the morphology of the cervical spine and Class II malocclusion by cone beam computerized tomography (CBCT). Ninety-three CBCT images belonging the file of Clinical Radiology, FOP-UNICAMP, of individuals of both sexes, without distinction of race and gender, with age between 18 and 35 years old. Evaluation of the morphology of the cervical spine was performend described by Sonnesen et al. (2007), the presence of fusion were observed, if there is no fusion was recorded as having normal morphology. Moreover, the morphology of the craniofacial complex was evaluated according to the measures proposed by Solow and Tallgren (1976). After collecting the data were tabulated for the validation of the method. It was evaluated by intraclass correlation coefficient (ICC) with a confidence interval of $95 \%$ and the association of normally distributed data were tested by Pearson correlation coefficient. It was observed an increase in the measurements of the craniofacial complex morphology in the group that presents fusion of the cervical column.
\end{abstract}

Key words: Cenvical spine, craniofacial morphology, Computed Tomography cone beam.

\section{Introduction}

Characteristics from skeletal malocclusions such as facial asymmetry, retrognathism or prognathism severe, can cause a muscle disorder affecting the stomatognathic system, which leads to a postural rehabilitation of the cervical vertebrae can cause an increase in cervical lordosis (Manfredini, 2012). Others show that the development of skeletal Class II comes from postural changes of the cervical spine (Solow, 1998; Leitão, 2000).

\section{Results and Discussion}

The results showed an intra- and inter-observer between satisfactory (ICC $\leq 0.4<0.75$ ) and excellent ( $I C C \geq 0.75$ ) for all evaluated measures. In addition, the average of the measurements when compared to the fusion and normal group was found higher for all variables of the relation cranio-sagittal and cranial base angle, as well as in almost all variables, which indicates the craniovertical relationship with the exception of variables $\mathrm{NL} / \mathrm{ML}, \mathrm{NSL} / \mathrm{ML}, \mathrm{NL} / \mathrm{VER}$ and gn-tgo-air. Moreover, Pearson coefficient showed no significant statistical correlation in any of the measurements for all the variables, when comparing the group that had fusion with the normal group.
Table 1. Average, standard deviation, maximum and minimum variable of the morphology of the craniofacial complex and the Pearson Correlation between fusion and normal group.

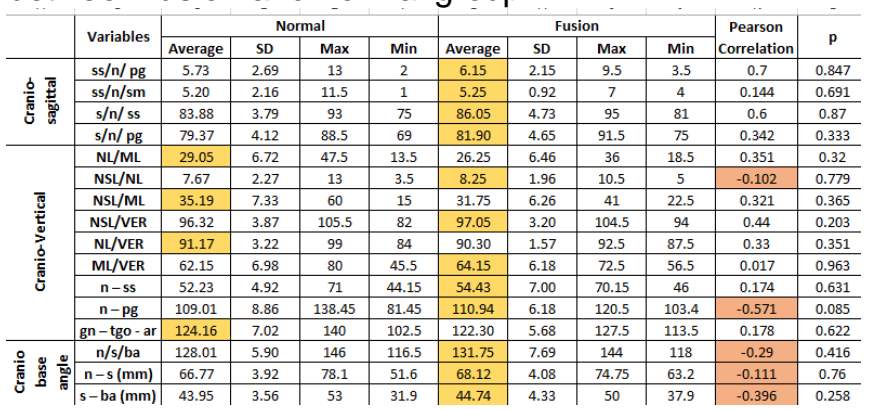

Conclusions

It was observed an increase in the measurements of the craniofacial complex morphology (craniosagittal relationship, craniovertical and cranio base angle) in the fusion group. A positive correlation in the variables of the cranio-sagittal and cranio-vertical relationships and negative correlation of the cranio base angle when compared the two groups.

\section{Acknowledgement}

We thank CNPq for financial support.

1. Sonnesen L, Pedersen CE, Kjaer I. Eur J Orthod 2007; 29(4):398403.

2. Solow B, Tallgren A. Am J Phis Anthrop 1976; 44(3):417- 